\title{
FUNCTIONS WITH PRESCRIBED LIPSCHITZ CONDITION
}

\author{
w. S. LOUD
}

1. Introduction. In this note we construct a class of functions each of which satisfies at every point a Lipschitz condition of prescribed order $\alpha(0<\alpha<1)$. The construction is based on a method given by Knopp [1 $]^{1}$ for construction of continuous nondifferentiable functions, and follows more particularly a construction of van der Waerden [2].

2. The construction. We first define a fundamental periodic function $g(t, h)$. The function $g(t, h)$ has period $2 h$ in $t$, equals zero for even multiples of $h$, equals one for odd multiples of $h$, and is linear between successive multiples of $h$. It is thus a saw-tooth function of $t$.

Now let a number $\alpha$ be given, where $0<\alpha<1$. Let $A$ be an integer for which

$$
2^{2 A(1-\alpha)}>2 .
$$

The function we are constructing is

$$
g(t)=\sum_{n=1}^{\infty} 2^{-2 A \alpha n} g\left(t, 2^{-2 A n}\right) .
$$

It will be shown that $g(t)$ satisfies for each value of $t$ a Lipschitz condition of order precisely $\alpha$.

\section{Proof of Lipschitz condition.}

THEOREM. The function $g(t)$ defined above satisfies for each value of $t$ a Lipschitz condition of order precisely $\alpha$. That is, there exist two positive constants $K_{1}$ and $K_{2}$ such that

(a) for any t and any $\Delta t$,

$$
|\Delta g|<K_{1}|\Delta t|^{\alpha}
$$

where $\Delta g=g(t+\Delta t)-g(t)$, and

(b) for any $t$ and for infinitely many, arbitrarily small $\Delta t$,

$$
|\Delta g|>K_{2}|\Delta t|^{\alpha} .
$$

Proof. We shall denote the $k$ th summand of the series for $g(t)$ by $g_{k}$. To prove assertion (a) we let $m$ be the integer such that

$$
2^{-2 A(m+1)}<\Delta t \leqq 2^{-2 A m} .
$$

Received by the editors May 6, 1950.

${ }^{1}$ Numbers in brackets refer to the bibliography at the end of the paper. 
Since the slope of the linear portions of $g_{k}(t)$ is $\pm 2^{2 A k(1-\alpha)}$,

$$
\left|\Delta g_{k}\right| \leqq 2^{2 A k(1-\alpha)}|\Delta t| \leqq 2^{2 A k(1-\alpha)-2 A m} \quad(k \leqq m) .
$$

Since the maximum oscillation of $g_{k}(t)$ is $2^{-2 A \alpha k}$,

$$
\left|\Delta g_{k}\right| \leqq 2^{-2 A \alpha k} \quad(k>m) \text {. }
$$

Combining the above,

$$
\begin{aligned}
|\Delta g| & \leqq \sum_{k=1}^{m} 2^{-2 A m} 2^{2 A(1-\alpha) k}+\sum_{k=m+1}^{\infty} 2^{-2 A \alpha k} \\
& \leqq \frac{2^{2 A(1-\alpha)-2 A \alpha m}}{2^{2 A(1-\alpha)}-1}+\frac{2^{-2 A \alpha(m+1)}}{1-2^{-2 A \alpha}} .
\end{aligned}
$$

Now $|\Delta t|^{\alpha}>2^{-2 A \alpha(m+1)}$. Therefore

$$
\frac{|\Delta g|}{|\Delta t|^{\alpha}}<\frac{2^{2 A}}{2^{2 A(1-\alpha)}-1}+\frac{1}{1-2^{-2 A \alpha}}
$$

which proves assertion (a).

To prove assertion (b) we use a lemma on geometrical progressions.

LEMмA. If the ratio in a geometric progression is positive and less than $1 / 2$, and the first term is 1 , then the first term exceeds the sum of the remaining terms by at least

$$
\frac{1-2 r}{1-r}
$$

Proof of Lemma. If the progression is infinite, the sum of the terms following the first is $r /(1-r)$, and if the progression is finite the sum of the terms following the first is less than this. If $r<1 / 2$, $r /(1-r)<1$, so that the first term, 1 , exceeds the sum of the remaining terms by at least

$$
1-r /(1-r)=(1-2 r) /(1-r) .
$$

Turning now to the proof of (b), for a given $t$ let $\Delta t$ be equal numerically to

$$
2^{-2 A(m+1)}
$$

and in a direction so as not to include a multiple of $2^{-2 \Lambda m}$, where $m$ is any positive integer. For this $\Delta t$,

$$
\Delta g_{k}=0
$$


while

$$
\Delta g_{k}= \pm 2^{2 A k(1-\alpha)} 2^{-2 A(m+1)} \quad \text { if } k \leqq m .
$$

Thus

$$
\begin{aligned}
\Delta g & =2^{-2 A(m+1)}\left[ \pm 2^{2 A m(\alpha-1)} \pm 2^{2 A(m-1)(1-\alpha)} \pm \cdots \pm 2^{2 A(1-\alpha)} \mid\right. \\
& =2^{-2 A-2 A m \alpha}\left[ \pm 1 \pm 2^{-2 A(1-\alpha)} \pm 2^{-4 A(1-\alpha)} \pm \cdots\right]
\end{aligned}
$$

Now since $A$ was chosen so that $2^{-2 A(1-\alpha)}<1 / 2$, we may apply the lemma and conclude that

$$
|\Delta g|>2^{-2 A-2 A m \alpha} \frac{1-2^{-2 A(1-\alpha)} \cdot 2}{1-2^{-2 A(1-\alpha)}} .
$$

Now $|\Delta t|^{\alpha}=2^{-2 \Delta m \alpha-2 \Delta \alpha}$. Therefore

$$
\frac{|\Delta g|}{|\Delta t|^{\alpha}}>2^{-2 A(1-\alpha)} \frac{1-2 \cdot 2^{-2 A(1-\alpha)}}{1-2^{-2 A(1-\alpha)}},
$$

and since this is true for any $m$, assertion (b) is proved.

It should be remarked that for any $\alpha$ in the range $0<\alpha<1$ the function $g(t)$ is continuous and nondifferentiable.

\section{BIBLIOGRAPHY}

1. K. Knopp, Ein einfaches Verfahren zur Bildung stetiger nirgends differenzierbar Funktionen, Math. Zeit. vol. 2 (1918) pp. 1-26.

2. B. L. van der Waerdan, Ein einfaches Beispiel einer nicht-differenzierbaren stetigen Funktion, Math. Zeit. vol. 32 (1930) pp. 474-475.

UNIVERSITY OF MINNESOTA 